\title{
Transport mechanisms inside the turbulent wake of a bluff body
}

\author{
Eliott VARON ${ }^{1}$, Nicolas MAZELLIER ${ }^{1}$, Azeddine KOURTA ${ }^{1}$ \\ 1. Univ. Orléans, INSA-CVL, PRISME \\ 8 rue Léonard de Vinci 45072 Orléans, France
}

\begin{abstract}
:
Based on the recent work of Stella et al. on the bidimensional (2D) flow behind a ramp [11, 10], the present paper aims at analysing the entrainment phenomenon in a three-dimensional (3D) turbulent wake downstream of a bluff-body. Numerical and experimental data of different configurations of the Ahmed body are investigated. This study is part of a framework on the aerodynamic drag reduction for ground vehicles by defining proper and realistic control laws derived from the entrainment.
\end{abstract}

Key words: turbulent shear flow, aerodynamics, bluff body, experiment, flow control

\section{Introduction}

Transport industry and more especially road transport remains one of the main contributors of energy consumption and pollutant emissions. To improve this negative situation, one way is to use active flow control to reduce drag. Vehicle wakes are characterized by both large pressure drag and coherent structures $[9,5,2,7]$. In addition, their 3D features, which contribute for large part of performance losses, make the physical mechanisms at play even more complex.

In this study, we consider the square back Ahmed body [8, 6]. This bluff body is characterized by an abrupt geometry change at the rear of the model that generates the vortices. These vortices interact with each other and lead to the formation of a large recirculation zone. This region is bounded by the shear layers emanating from the edges of the base of the model. Moreover, the boundary layer developing on the ground modifies the lower shear layer. This interaction alters the vortex emission in the wake and thus the pressure distribution on the base of the model responsible for an important part of the pressure drag. The flow of the turbulent near wake has complex dynamics like a bimodal behavior [3]. Time dependent data show that two asymmetric topologies, called reflectional symmetry-breaking (RSB) modes, coexist in the wake of the model but time averaging leads to a symmetric flow $[4,12]$. The bimodality does not seem to interfere with the other periodic modes that can be detected by means of spectral analysis. As far as the periodic modes are concerned, many authors agree with the identification of the low-frequency modes associated with vortex shedding, both from the left-/right-side planes and from the roof and the floor of the vehicle [12]. It is thus interesting to focus on the large-scale effects on the wake dynamics. We aim therefore at investigating turbulent entrainment at large-scale in the wake behind the Ahmed body to verify if the results obtained by Stella et al. [11, 10] for a 2D flow over a descending ramp can be confirmed for a 3D fully turbulent flow. The idea consists in finding relations between mean-field features of the wake like the base suction and the role of mass or momentum transfer. The final goal is to identify the unsteady phenomena into the wake of the square back Ahmed body through these relations, with a view to the development of optimized flow control strategies. We choose the recirculation region interface (RRI) as the boundary through which entrainment will be estimated.

\section{Experimental setup}

Tests are performed in the subsonic wind tunnel of the PRISME laboratory at the university of Orléans [12]. The square test section is $2 \mathrm{~m}$ by $2 \mathrm{~m}$ and $5 \mathrm{~m}$ long. The free-stream turbulence level is less than $0.4 \%$. The square back Ahmed body has the original dimensions [1]: $L=1044 \mathrm{~mm}, H=288 \mathrm{~mm}, W=389 \mathrm{~mm}$. The Reynolds number $R e_{H}$ is based on the body height. The model is mounted with a ground clearance $G=50 \mathrm{~mm}$ above a $3 \mathrm{~m}$ by $2 \mathrm{~m}$ aluminium plate with an ellipsoidal leading edge in order to be isolated from the wind tunnel boundary layer [see Fig. 1(a)]. This results in a blockage ratio lower than $3 \%$. The rear part of the plate is equipped with a $3^{\circ}$ inclined flap in order to suppress the pressure gradient effect in the test section. Five freestream velocities are investigated: from $U_{\infty}=20 \mathrm{~m} / \mathrm{s}$ $\left(R e_{H}=3.9 \times 10^{5}\right)$ to $U_{\infty}=40 \mathrm{~m} / \mathrm{s}\left(R e_{H}=7.7 \times 10^{5}\right)$.

The Ahmed body is linked to a six-components strain gauge balance through its feet to evaluate the drag and lift coefficients. The mean pressure field over the rear surface is measured by means of 32 pressure taps connected to one MicroDAQ pressure scanner inside the body through 
30-cm vinyl tubes. The electronic device applies a $0.5 \mathrm{~s}-$ moving average filter during the acquisition. The pressure field dynamics are captured by five Kulite sensors. Pressure sensors positions are given in Fig. 1(a) (red: microDAQ, blue: Kulite). Spatial characterisation of the wake of the Ahmed body was carried out using stereoscopic Particule Image Velocimetry (stereo PIV) system. As shown by Fig. 1(b), two kinds of planes are successively investigated: six vertical XZ-planes and five horizontal XY-planes. Data are simultaneously acquired during six runs lasting 5 minutes each for each freestream velocity and for each PIV plane by the previously presented sensors. The sampling frequency are $500 \mathrm{~Hz}$ (balance and Kulite), $50 \mathrm{~Hz}$ (microDAQ) and $1 \mathrm{~Hz}$ (stereo PIV).

The profiles of the boundary layer upstream of the bluff body, of the underflow and of the upper boundary layer upstream of the flow separation are also measured by a one-component hot-wire probe.

\section{Entrainment in a 3D wake}

The mass entrainment through a $2 \mathrm{D}$ interface $\mathcal{I}$ is

$$
\dot{m}=\int_{\mathcal{I}} \rho \boldsymbol{V}(s) \cdot \boldsymbol{n}(s) \mathrm{d} s,
$$

where $\mathrm{d} s$ is the elementary length at the position $s$ and $\boldsymbol{n}$ is its unit normal vector. Figure 2 shows the mass transfer through the RRI (where $U=0 \mathrm{~m} / \mathrm{s}$ ) detected in three different horizontal planes for both RSB modes and the mean flow. To better highlight the mass injected into the recirculation bubble and the mass ejected from it, the mass entrainment is integrated by parts between the positions of the $3 \mathrm{D}$ toroidal vortex detected in the planes. First, the mass transfer is clearly unbalanced between the upper part of the wake and its lower part. Second, the BSR modes seems to stress this unbalanced transfer. To have a better insight, we need to consider the $3 \mathrm{D}$ wake.

Using the wall-pressure measurements, conditional averaging is applied to the stereo PIV data (components velocity and Reynolds tensor) to obtain sliced RSB modes for each PIV plane. The full 3D RSB modes are then reconstructed by interpolation. Equation 1 is also used to analyse the mass transfer in the 3D fully turbulent near wake. The local mass transfer across the 3D interface $\mathcal{I}$ is now

$$
\mathrm{d} \dot{m}(s)=\rho \boldsymbol{V}(s) \cdot \boldsymbol{n}(s) \mathrm{d} S,
$$

where $\mathrm{d} S$ is the elementary surface at the position $s$ and $\boldsymbol{n}$ is its unit normal vector [see Fig. 1(c)]. A detailed analysis will be presented during the conference.

\section{Conclusions:}

Experimental studies have been done to characterize the 3D fully turbulent near wake behind the squareback Ahmed body, especially to analyse a possible relation between the mass transfer through the developing shear layer and the bimodal behaviour.

Acknowledgement: This work was supported by the Agence Nationale de la Recherche (ANR) through the Investissements d'Avenir program under the Labex CAPRYSSES Project (ANR-11-LABX-0006-01).

\section{References}

[1] S. Ahmed, G. Ramm, and G. Faltin. Some salient features of the time-averaged ground vehicle wake. In SAE Technical Paper, page 840300. SAE International, 1984.

[2] E. Bideaux, P. Bobillier, E. Fournier, P. Gilliéron, M. El Hajem, J. Y. Champagne, P. Gilotte, and A. Kourta. Drag reduction by pulsed jets on strongly unstructured wake: towards the square back control. Int. J. Aerodyn., 1(3-4):282-298, 2011.

[3] M. Grandemange, O. Cadot, and M. Gohlke. Reflectional symmetry breaking of the separated flow over three-dimensional bluff bodies. Phys. Rev. E, 86(3):035302, 2012.

[4] M. Grandemange, M. Gohlke, V. Parezanović, and O. Cadot. On experimental sensitivity analysis of the turbulent wake from an axisymmetric blunt trailing edge. Phys. Fluids, 24(3):035106, 2012.

[5] E. Guilmineau. Computational study of flow around a simplified car body. J. Wind Eng. Aerodyn., 96(6):1207-1217, 2008.

[6] S. Krajnović and L. Davidson. Flow around a simplified car, part 2: Understanding the flow. J. Fluids Eng, 127(5):919-928, 2005.

[7] J. W. McNally, F. S. Alvi, N. Mazellier, and A. Kourta. Active flow control on an Ahmed body - An experimental study. In 53rd AIAA Aerospace Sciences Meeting. Kissimmee, Florida. AIAA SciTech Forum, 2015.

[8] M. Rouméas, P. Gilliéron, and A. Kourta. Analysis and control of the near-wake flow over a square-back geometry. Comput. Fluids, 38(1):60-70, 2009.

[9] A. Spohn and P. Gilliéron. Flow separations generated by a simplified geometry of an automotive vehicle. In IUTAM Symposium: unsteady separated flows, pages 8-12, 2002. 


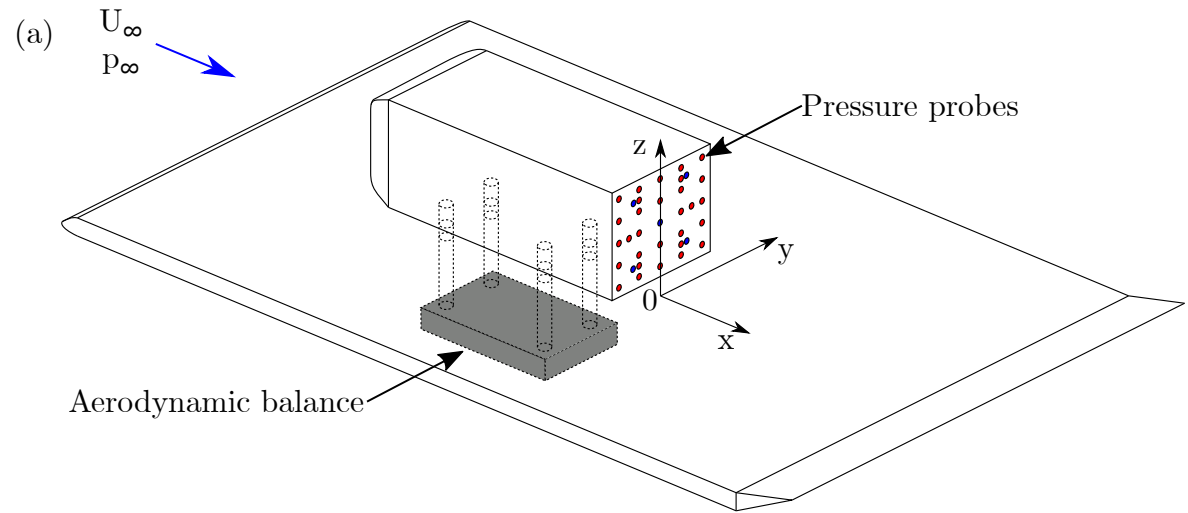

(b)

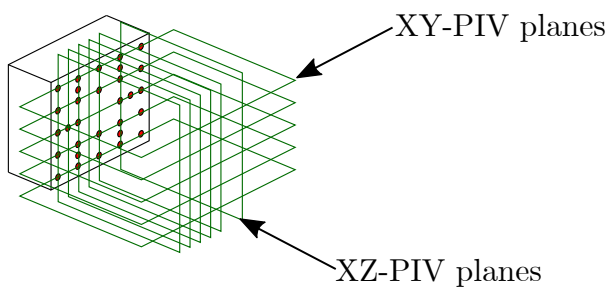

(c)

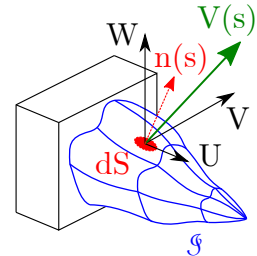

Figure 1: Experimental setup: (a) aerodynamic balance and pressure taps (red: microDAQ, blue: Kulite) and (b) stereoPIV planes positions. (c) Example of an elementary surface $d S$ with its normal vector $\boldsymbol{n}$ and its local velocity $\boldsymbol{V}(U, V, W)$ over an interface $\mathcal{I}$.
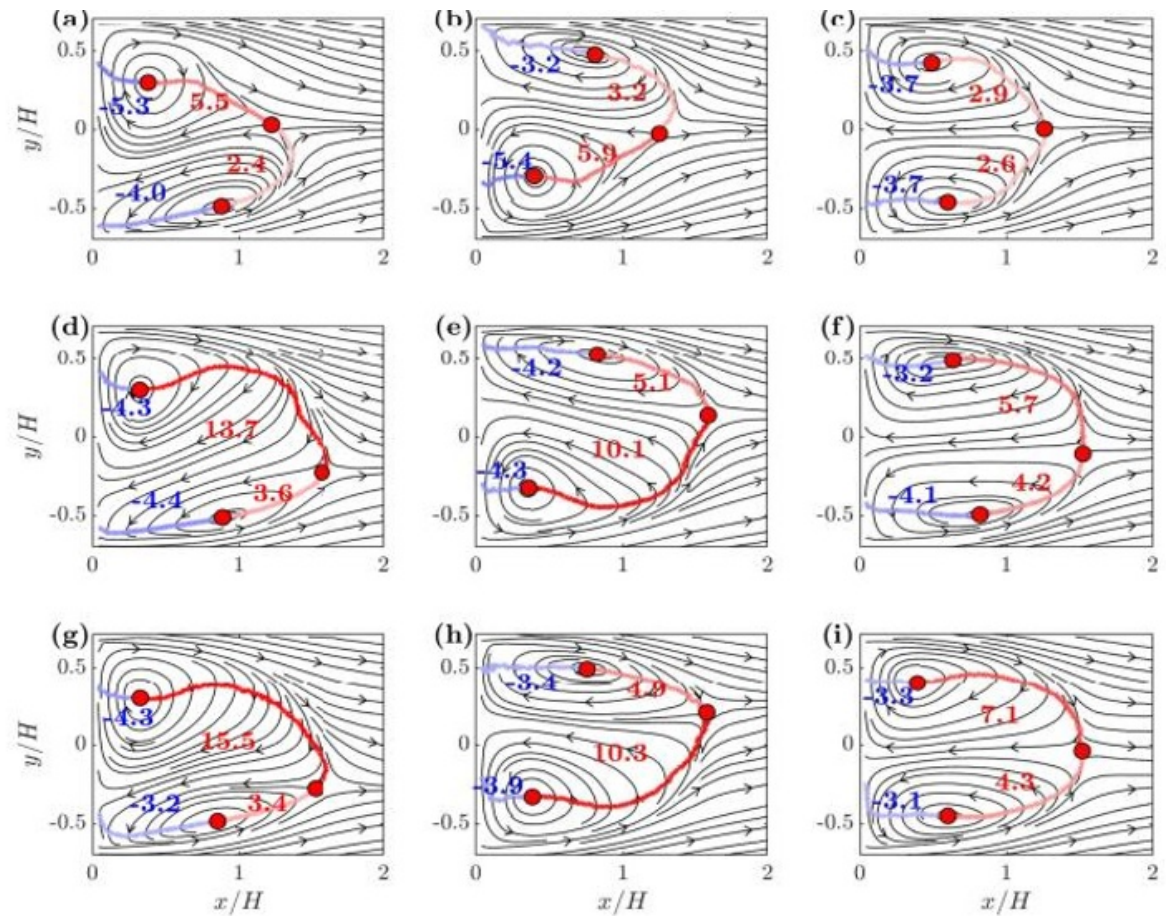

Figure 2: Mass transfer between the detected positions of the 3D toroidal vortex (red dots) on the RRI at $z / H=1.1$ (top), $z / H=0.7$ (medium) and $z / H=0.3$ (bottom) for the P-mode (left), the N-mode (middle) and the mean flow (right). Red values are injected mass into the recirculation bubble, whereas blue values are ejected mass. $\operatorname{Re}_{H}=7.7 \times 10^{5}$.

[10] F. Stella, N. Mazellier, P. Joseph, and A. Kourta. Mass entrainment-based model for separating flows. Phys. Rev. Fluids, 3:114702, Nov 2018.

[11] F. Stella, N. Mazellier, and A. Kourta. Scaling of separated shear layers: an investigation of mass entrainment. J. Fluid Mech., 826:851-887, 2017.
[12] R. Volpe, P. Devinant, and A. Kourta. Experimental characterization of the unsteady natural wake of the full-scale square back Ahmed body: flow bistability and spectral analysis. Exp. Fluids, 56(5), 2015. 